\title{
The Effect of Rendering Intents on Color Quality of Digital Printing
}

\author{
Adel Mohamed M. Khodeir ${ }^{1}$ \\ Faculty of Applied Arts, Helwan University, Cairo, Egypt \\ adel.ibr@cas.edu.om
}

\begin{abstract}
The adjustment of color to achieve an acceptable match between the displayed and digital printed document is an important task in prepress. In order to achieve such match color management systems are used, these systems implement standards established by the International Color Consortium (ICC). A key step of the color management process is the calibration of display and output devices, the definition of the relationship between the native color space of the device and a standard device-independent color space. In this work the usability of the ICC color management standard was investigated in case of flat panel LCD display calibration.
\end{abstract}

Keywords: Color Management, Lithography, Printing Quality, and Rendering Intent.

\section{Introduction}

The issue of managing and controlling color, from scanners to monitors to digital printers/proofers and finally to lithographic printing presses, is a serious one for the printing and imaging industries. Accurate color control is vital in order to have a predictable quality of final product, whether it be printed on a substrate or displayed in an image editing program or a Web browser, and ultimately to satisfy

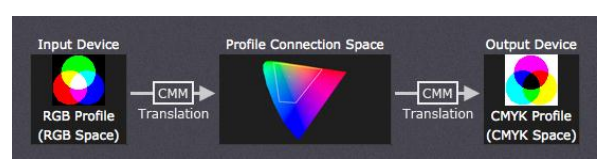

Fig. 1: The structure of normal Desktop Publish system in normal printing condition.

Customer expectations. The costs involved in controlling color for the designer/publisher/printer are both soft (time spent by employees during pre publication, hard or soft proofing, film or plate output for printing presses, and on press), and hard (wasted ink and substrate used in make-ready, as well as time wasted in jobs that do not meet final customer specifications).

Great strides have been made in the printing industry in recent years toward applying scientific methods in the pressroom, as well as in the prepress area. Printing has become much more science than art and every company, especially printing and media companies, must have a presence on the World Wide Web. This makes controlling agreement between displayed and printed color more important than ever. (Fig. 1)

\section{Gamut and Rendering Intents}

When working in a color managed workflow with ICC profiles, it is important to remember the "Three Cs" of Color Management. These are calibration, characterization and conversion. Calibration is the process of ensuring that the device in question conforms to a specified state or 
condition. The profiling process characterizes the color behavior of the properly calibrated device. Conversion is what is needed for applications to convert between different device color spaces. (Fig. 2) This is required in order to display or print scanned images or to preview (soft proof)

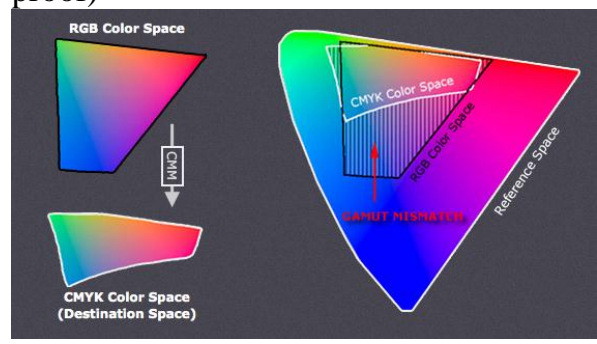

Fig. 2: Transformation from RGB to CMYK color gamut

color spaces and Gamut mismatch.

What the printed image will look like on a calibrated and characterized output device. Varying degrees of support for these conversions are available in Adobe Photoshop, InDesign, Illustrator, QuarkXPress, and PageMaker. Of these, Photoshop CS6 and CC are the most advanced and the easiest to manipulate and probe. In addition, there is some degree of support at the operating system level from Mac OS 9.2.2 (or earlier), Mac OS $\mathrm{X}$ and Windows. (Fig. 3)

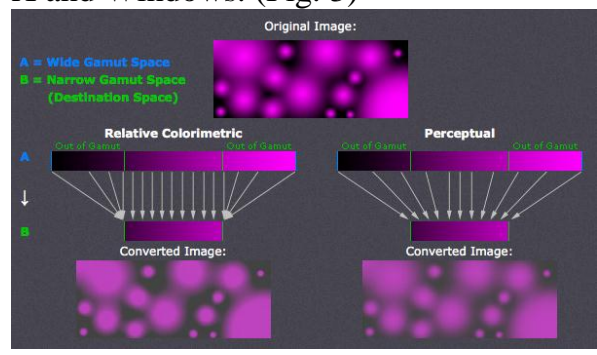

Fig. 3: Comparison between Relative and Perceptual rendering intents.

Color space conversion is what happens when a color management module (CMM) translates the colour from a device's space to other space. This
Conversion requires approximates in order to preserve the image's most important color qualities. We should know how these values work can help to control how the photo may change hopefully maintaining the intended look or mood.

\section{The algorithm of Rendering Intents on Color Quality of Printing}

In this study we used the mathematical algorithm (CIE, 2004). The lightness is mapped using the following formula:

$$
L_{r}^{*}=\left(1-p_{c}\right) L_{o}^{*}+p_{c} L_{S}^{*}
$$

Where $\mathrm{L}^{*}{ }_{\mathrm{o}}$ is the original lightness, $\mathrm{L}_{\mathrm{r}}^{*}$ is the reproduction lightness, $\mathrm{L}_{\mathrm{S}}^{*}$ is the result of the original lightness being mapped using a sigmoidal function, and $\mathrm{pc}$ is a chroma-dependant weighting factor that depends on the Chroma of the original color, $\mathrm{C}^{*}$.

$$
p_{c}=1-\left(\left(C^{* 3}\right) /\left(C^{*} 3+5.10^{5}\right)\right)^{1 / 2}
$$

To calculate $\mathrm{L}^{*}$, a one dimensional lookup table (LUT) between original and reproduction lightness values is first set up on the basis of a discrete cumulative normal function $(\mathrm{S})$.

$S_{i}={ }_{n=0}^{n=i} \frac{1}{\sqrt{2}} e^{\frac{\left(\frac{100 n}{m} x_{0}\right)^{2}}{2}}$

Where $\mathrm{x}_{0}$ and $\Sigma$ are the mean and standard deviation of the normal distribution $\mathrm{n}$ respectively,

$\mathrm{i}=0,1,2 \ldots \mathrm{m}+1$ is the number of points used in Look Up Table. $S_{i}$ is the value of the cumulative normal function for $\mathrm{i} / \mathrm{m}$ percent.

\section{Gamut Mismatch \& Rendering Intent}

The translation stage attempts to create a best match between devices - even when seemingly incompatible. If the source device has a larger color gamut than the destination device, some of those colours will be outside the destination 
device's color space. These "out-of-gamut colors" occur with nearly every conversion and are called a colour gamut mismatch. Each time a color gamut mismatch occurs, the Color Management Module uses the rendering intent to determine the qualities of the image, which should prioritize. Common rendering intents include: relative and absolute colorimetric, saturation and perceptual. Every one of these types deals with one property of colour at the expense of the other rendering intents. (Fig. 4)

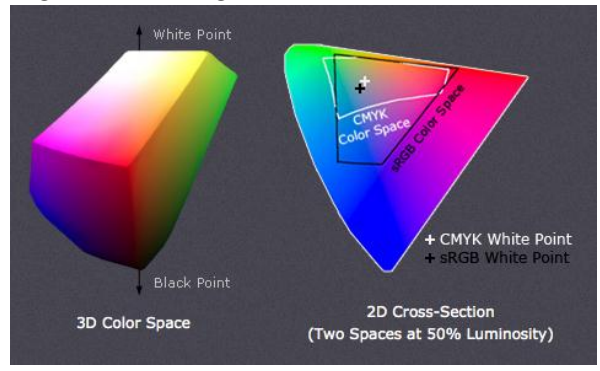

Fig. 4: 3D color space and 2D cross-section (Two spaces at 50\% Luminosity).

\section{Perceptual \& Relative Colorimetric Intents}

Perceptual and relative colorimetric rendering are probably the most useful conversion types for digital imaging. Every locations another priority on how they render colors within the gamut mismatch region. (Fig. 5)

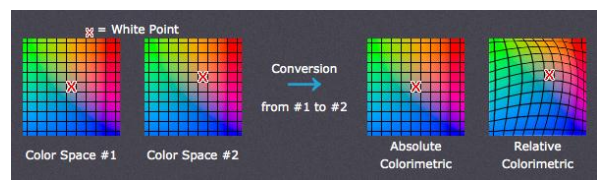

Fig. 5: The transformation of color gamut using absolute Colorimetric and Relative Colorimetric Rendering Intents.

Relative colorimetric maintains a near exact relationship between in gamut colors, whatever this clips out of gamut colours. In the other hand, perceptual render- ing preserves some relationship between out of gamut colors, even if those results are inaccuracies for gamut colours.

\section{Absolute Colorimetric Intents}

Absolute is similar to relative colorimetric in that it preserves in gamut colors and clips which are out of gamut, but they vary in how each calculate the white point. The white point is the coordination of the lightest white in a colour space. If one were to draw a line between the white and black points, this might pass via the most neutral colours.

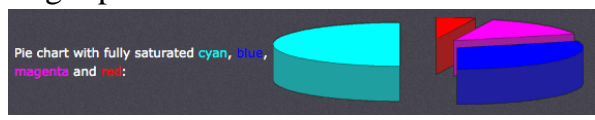

Fig. 6: Example of Pie color chart with fully saturated cyan, blue, magenta and red, in absolute colorimetric intents.

The location of this line often changes between color spaces. Relative colorimetric skews the colors throw the gamut so that the white point of one space aligns with that of the other, while the absolute colorimetric rendering intent preserves colours exactly

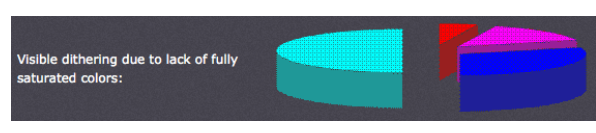

Fig. 7: the same example showing visible dithering due to lack of fully saturated colors.

(Without regard to changing white point). To illustrate this, the example Figures 6 and 7 shows two theoretical spaces that have similar gamuts, but with different white points.

\section{Saturation Intents}

Saturation rendering intent tries to preserve saturated colours is very useful 
when trying to retain color purity in computer graphics when converting into a wider colour space. If the source RGB space device contained pure (saturated) colours, then saturation intent ensures that those colors will remain saturated in the new color space - even if this causes the colors to become relatively more extreme.

Maintaining color saturation may come at the expense of changes in hue and lightness, which is usually an unacceptable result for image reproduction. This is often acceptable for computer graphics such as pie charts.

Another use for saturation intent is to avoid visible dithering when printing computer graphics on inkjet printers. Some dithering may be unavoidable as the inkjet printing never have an ink to match every colour, whatever the saturation intent can reduce those cases where dithering is sparse because the color is very close to being pure.

\section{Conclusion}

This study has evaluated the performance of selected gamut mapping in order to
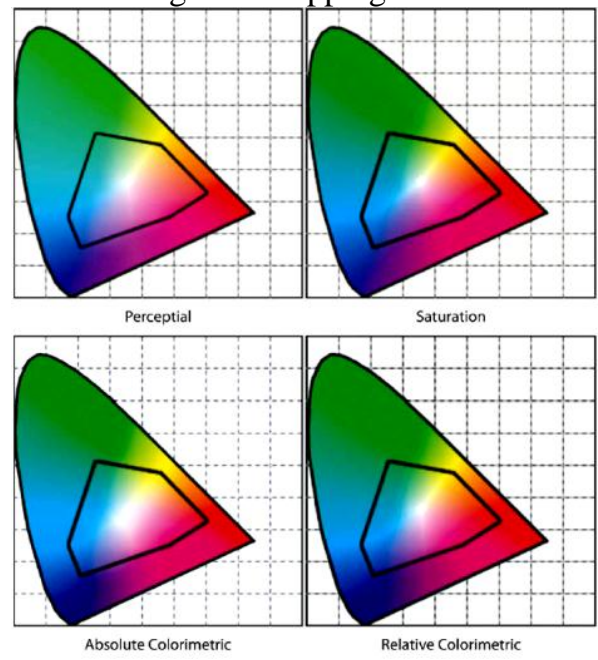

Fig. 8: A comparison Charts showing the different color gamuts for each method of rendering intents.

Answer a question, this question which should be asked; which is the best rendering intent for digital printing? In general, perceptual and relative colorimetric are best suited for digital printing because they aim to preserve the same visual appearance as the original. The decision about when to use each of these depends on image content and the intended purpose. Images with intense colors (such as bright sunsets or well-lit floral arrangements) will preserve more of their color gradation in extreme colors using perceptual intent.

On the other hand, this may come at the expense of compressing or dulling more moderate colors. Images with more subtle tones (such as some portraits) often stand to benefit more from the increased accuracy of relative colorimetric (assuming no colors are placed within the gamut mismatch region). Perceptual intent is over the safest bet for general and batch use, otherwise we know specifics about every photo. (Fig. 8)

This study, which has used a high number of test images, shows also the accuracy of using various test image characteristics to get reliable results, as they are image dependent.

\section{References}

1. Paul D. Fleming and Abhy Sharma, Color Management with ICC Profiles: Can't live without it so learns to live with it. West Michigan University, MI: Kalamazo, May 2008.

2. Akos Borbely, Accurate color Reproduction in Prepress, Institute of Media Technology, Budapest Technology, Hungary, March 2003. 
3. A Sharma and P D Fleming, "Evaluating the Quality of Commercial ICC Color Management Software", Presented at TAGA Annual Technical Conference.

4. Fabienne Dugayand and Lionel Chagas, "Perceptual evaluation of colour gamut mapping algorithms ", The Norwegian Color Research Laboratory - Høgskolen i Gjøvik (Norway)
5. Adel M. Khodeir, Using Color Management Systems in DTP, to achieve Color Matching, with application on Commercial Lithographic Press. Helwan University, Cairo, April 2004.

6. A Learning community for Photographers, Colour Space Conversion, Cambridge in Colour, Cambridge, UK 2012 